\title{
Time-dependent approach to high-resolution spectroscopy and quantum dynamics of Rydberg atoms in crossed magnetic and electric fields
}

\author{
Xiao-Min Tong and Shih-I Chu \\ Department of Chemistry, University of Kansas, and Kansas Center for Advanced Scientific Computing, Lawrence, Kansas 66045
}

(Received 16 December 1998; published 1 February 2000)

\begin{abstract}
We present a general time-dependent approach for efficient and accurate treatment of high-resolution spectrocopy and quantum dynamics. The procedure is applied to an ab initio time-dependent study of threedimensional Rydberg $\mathrm{H}$ atoms in crossed magnetic and electric fields with spectral accuracy. Good agreement with the high-resolution experimental photoabsorption spectrum is obtained line by line, including a regime well above the Stark saddle point. We have further explored the physical origin of several unexplored perplexing phenomena in the higher-energy regime observed in the experiment.
\end{abstract}

PACS number(s): 32.60.+i, 32.70.Cs, 32.80.Dz, 32.80.Rm

The subject of Rydberg $\mathrm{H}$ atoms in crossed magnetic and electric fields has attracted considerable attention in the last several years both experimentally [1-3] and theoretically [4-10]. This is a nonseparable and nonintegrable system with three degrees of freedom and great complexity. It is known that the hydrogen atom in a pure magnetic field undergoes a transition from regular to chaotic motion at energies below the ionization threshold [11]. In contrast, the hydrogen atom in a pure electric field is separable in parabolic coordinates and represents an example of a regular open system. Thus a crossed-field system allows a study of the transition between these two extreme situations. In addition to its fundamental importance, the crossed-field problem is also relevant to a number of outstanding issues in celestial mechanics [12], atomic and molecular physics, solid-state physics [13], nuclear physics [14], and astrophysics [15].

The high-resolution photoabsorption spectrum of Rydberg $\mathrm{H}$ atoms in crossed fields performed by Wiebusch et al. [1] revealed several novel and complex features. First, they reported long-living states exist well above the Stark saddle energy. Second, the spectral line positions can be accounted for reasonably well by the second-order perturbation theory (SOPT) $[4,5]$ for the regimes below and somewhat above the Stark saddle energy. This suggests that the purported transition from order to chaos may be somewhat delayed or suppressed. However, the SOPT deviates more and more from the experimental data as the principal quantum number $n$ increases, and it fails completely in the higher-energy regime (above $-250 \mathrm{~cm}^{-1}$ ). Another salient feature of the experimental spectrum shown in Fig. 2 of Ref. [1] is the apparently "simpler"' spectrum in the higher-energy regime (an energy beyond $-220 \mathrm{~cm}^{-1}$ ), where one would expect to see chaotic or at least denser spectral feature. To date, there is no detailed nonperturbative three-dimensional (3D) quantum study that has been performed to explain the origin of these observed perplexing phenomena [1].

In the theoretical investigation of Rydberg atoms in static external fields, most quantum calculations so far employed time-independent methods, namely, perturbation theory for low-lying states [4,5] and the variational basis-set-expansion method for higher-lying Rydberg states $[6,8]$. The latter often involves the diagonalization of matrices of considerably larger size. Classical approaches $[9,10]$ have provided useful insights regarding Rydberg atom nonlinear dynamics, but quantum mechanics is required for an explanation of delicate spectral features. An alternative possible quantal approach is the time-dependent method, which numerically integrates the time-dependent Schrödinger equation in space and time. The time-dependent approach has several appealing features not shared by the time-independent approach. For example, it provides a direct probe of the time-resolved Rydberg atom dynamics. It also allows the study of the classical-quantum correspondence through the recurrence spectrum obtained by the Fourier transformation of the photoabsorption spectrum in the time domain. Although there are time-dependent treatments of lower-dimensional Rydberg systems [16], to our knowledge no time-dependent method has been developed and applied successfully to the study of high-resolution photoabsorption spectrum of realistic 3D Rydberg systems in external fields, particularly in the classically chaotic regime. The difficulties stem from the fact that Rydberg atom problems involve very large spatial extent, long-time propagation of the time-dependent Schrödinger equation, and the existence of the Coulomb singularity at the origin. All of these cannot be adequately treated by the traditional timepropagation methods [17] using equal-spacing spatial grid discretization techniques.

The motivation of this Rapid Communication is twofold. First we present a time-dependent nonperturbative approach to overcome the above-mentioned difficulties. The procedure is based on the extension of a recent development of the generalized pseudospectral time-dependent method [18], which allows for nonuniform spatial grid discretization and long-time propagation of the Schrödinger equation with both accuracy and efficiency. The method was recently applied successfully to a study of the photoabsorption spectrum of Rydberg $\mathrm{H}$ atoms in a static magnetic field (a 2D problem), and reproduced the experimental spectrum line by line well within the classically chaotic regime [19]. In this paper we extend the method to the $3 \mathrm{D}$ crossed-field problem. We note the extension from the $2 \mathrm{D}$ to $3 \mathrm{D}$ Rydberg system is by no means straightforward. Due to the complete symmetry breaking, the 3D system possesses no good quantum numbers. Indeed, the extreme difficulty in performing highprecision 3D Rydberg calculations with spectral accuracy has delayed a detailed analysis of the experimental spectrum 
[1] for nearly a decade. In this paper, we present a timedependent procedure which allows a precise calculation of the photoabsorption spectrum of Rydberg $\mathrm{H}$ atoms in strong cross fields, with results in good agreement with the experimental data [1]. Further we explore in detail the physical origin of the experimentally observed phenomena well above the Stark saddle point, which to our knowledge has not been understood so far.

It is known [20] that the photoabsorption cross section $\sigma(\omega)$ can be determined by the Fourier transform of the dipole autocorrelation function $C(t) \equiv\langle\psi \mid \psi(t)\rangle$,

$$
\sigma(\omega)=2 \pi \alpha a_{0}^{2} \omega \int d t e^{i E t} C(t)
$$

with $|\psi\rangle=|\psi(0)\rangle=\hat{\mu}\left|\phi_{0}\right\rangle$. Here $\omega$ is the light frequency, $\alpha$ the fine-structure constant, $a_{0}$ is the Bohr radius, $\hat{\mu}$ is the transition dipole operator, $\phi_{0}$ is the initial bound state (with energy $\left.E_{0}\right), E=E_{0}+\omega$, and $|\psi(t)\rangle$ satisfies the timedependent Schrödinger equation, in atomic units

$$
i \frac{\partial}{\partial t}|\psi(t)\rangle=\hat{H}|\psi(t)\rangle
$$

where $\hat{H}$ is the total Hamiltonian which generates the finalstate eigenfunction. Equation (1) is the central theme of the time-dependent approach of spectroscopy: it connects the time-resolved dynamics as reflected by the time autocorrelation function $C(t)$ on one hand, to the frequency-resolved cross section $\sigma(\omega)$ on the other. Heller [20] pioneered the time-dependent approach for the treatment of classical dynamics, and the corresponding quantum spectral features in the photoabsorption dynamics of small polyatomic molecules. In the context of Rydberg atom dynamics, the timedependent approach has been extended to the study of shorttime electronic wave-packet propagation in crossed fields [21], and the gross features of photoabsorption spectrum in magnetic fields [22], using classical trajectories. To date, with the exception of our recent work on the magnetic fields [19], the time-dependent approach [Eq. (1)], has not been extended to the study of high-resolution photoabsorption spectrum and long-time dynamics of Rydberg atoms in external fields due to the computational challenges indicated earlier. Below we discuss the extension of a time-dependent procedure $[18,19]$ for precision calculation of the photoabsorption spectrum of Rydberg $\mathrm{H}$ atoms in strong crossed fields.

The Hamiltonian of the hydrogen atom in crossed magnetic $\vec{B}(\| \hat{z})$ and electric $\vec{F}(\| \hat{x})$ fields can be written as, in atomic units,

$$
H=-\frac{\nabla^{2}}{2}-\frac{1}{r}+\frac{1}{2} \gamma \hat{L}_{z}+\frac{1}{8} \gamma^{2}\left(x^{2}+y^{2}\right)-F x
$$

where $\mathrm{F}$ is the electric-field amplitude, $\gamma$ is the magnetic field $B$ amplitude in units of $B_{0}=2.35 \times 10^{5} \mathrm{~T}$, and $\hat{L}_{z}$ is the $z$ component of the orbital angular momentum. To solve the time-dependent Schrödinger equation [Eq. (2)], we first par-

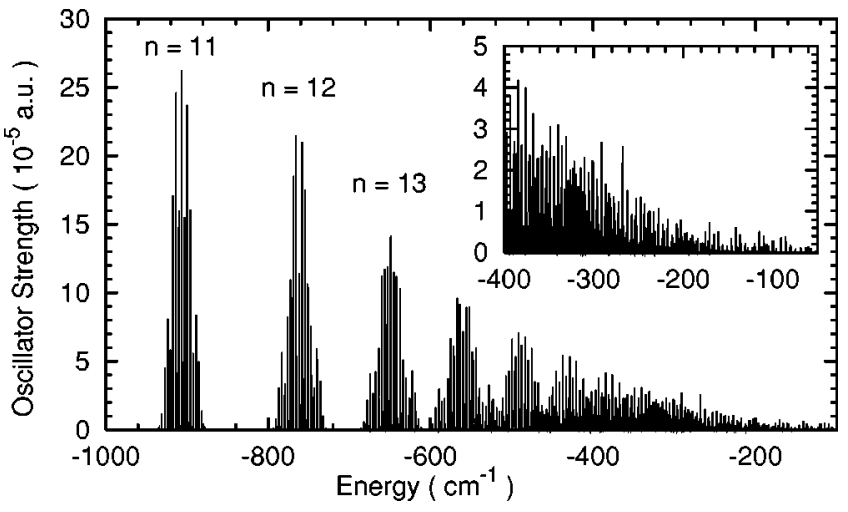

FIG. 1. Calculated oscillator strength of atomic $\mathrm{H}$, initially prepared in the $2 p_{0}$ state, in crossed magnetic $(B=6 \mathrm{~T})$ and electric $(F=2975 \mathrm{~V} / \mathrm{cm})$ fields.

tition the total Hamiltonian into three separate terms $H$ $=H_{o}+V_{1}+V_{2}$, where $H_{o}$ is the unperturbed atomic hydrogen Hamiltonian, and

$$
V_{1}=\frac{1}{2} \gamma \hat{L}_{z}, \quad V_{2}=\frac{1}{8} \gamma^{2}\left(x^{2}+y^{2}\right)-F x .
$$

The wave function is propagated by the second-order splitoperator method in spherical coordinates and in the energy representation [18]

$$
\begin{aligned}
\psi(\mathbf{r}, t+\Delta t)= & e^{-i H_{o} \Delta t / 2} e^{-i V_{1} \Delta t / 2} e^{-i V_{2} \Delta t} \\
& \times e^{-i V_{1} \Delta t / 2} e^{-i H_{o} \Delta t / 2} \psi(\mathbf{r}, t)+O\left(\Delta t^{3}\right) .
\end{aligned}
$$

To pursue the time propagation, we first express the total wave function in a partial-wave expansion [18]. The semiinfinite domian $[0, \infty]$ of the radial coordinate $r$ is transformed into a finite domain $\xi=[-1,1]$ by means of a suitable nonlinear algebraic mapping [18,23]. A generalized pseudospectral technique [23] is then extended to perform optimal radial grid discretization on the transformed Hamiltonian, allowing a denser mesh near the origin and at the same time taking into account the long-range interaction. The number of grid points needed is significantly (orders of magnitude) smaller than those required by the conventional equal-spacing discretization methods [17,24]; however, a considerably higher accuracy of the wave function can be achieved. The "energy" representation in Eq. (5) allows the elimination of the undesirable fast oscillating "high-energy" components, and the use of a substantially larger time step $\Delta t$. Further, each partial-wave wave-function component can be propagated independently under $H_{o}$, leading to a further significant reduction of the computational time needed. We refer the readers to Ref. [18] for numerical details of the procedure.

Figure 1 displays the global photoabsorption oscillator strength pattern from -1000 to $0 \mathrm{~cm}^{-1}$, corresponding to the excitation of atomic $\mathrm{H}$, initially prepared at the $2 p_{0}$ state, by linearly polarized light. The electric-field amplitude $F$ $=2,975 \mathrm{~V} / \mathrm{cm}$ and the magnetic field strength $B=6 \mathrm{~T}$ used in the calculation are the same as those employed in the experiment [1]. Figure 1 shows that there is no $n$ mixing for 


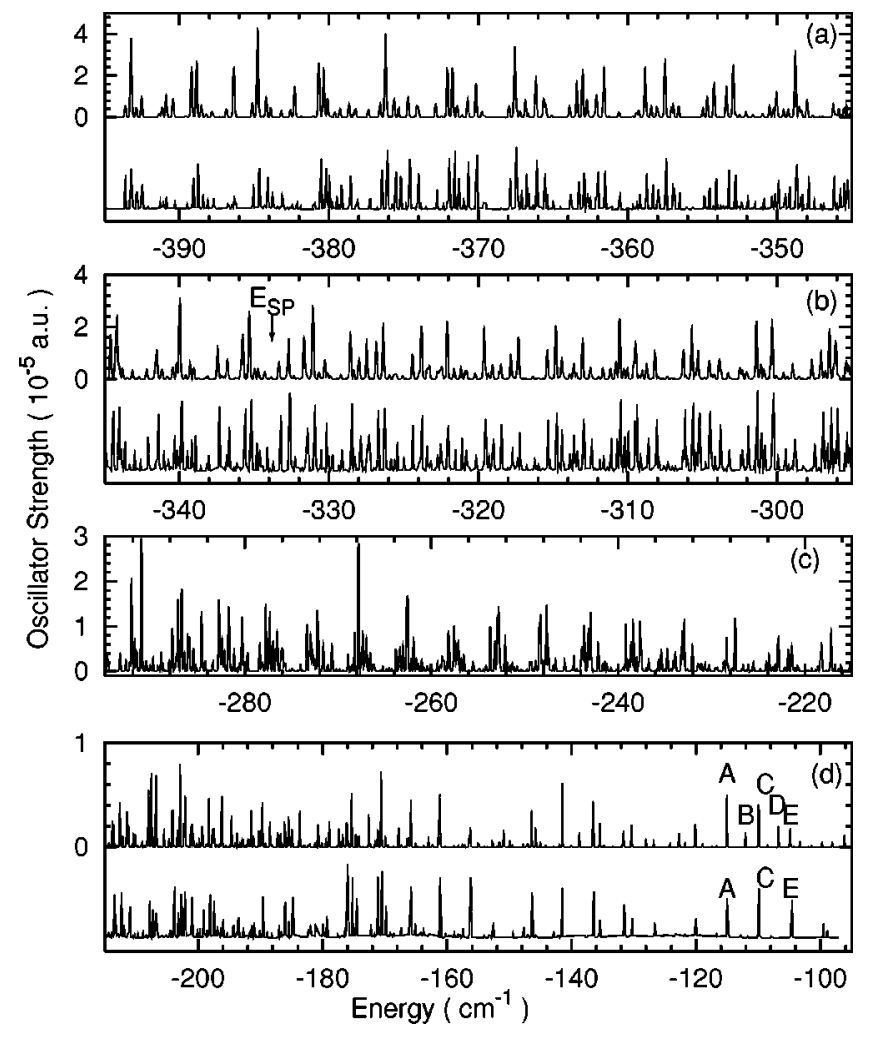

FIG. 2. Comparison of the theoretical oscillator strength spectrum (upper panels) and the experimental photoabsorption spectrum (lower panels) for the $\Delta m=0$ Balmer transition from the initial $2 p_{0}$ state to $m=0$ even-parity Rydberg states, covering the energy range from -395 to $-100 \mathrm{~cm}^{-1}$. The experimental intensity scale is in arbitrary units. In (c), only the calculated spectrum is available for presentation.

low-lying Rydberg states $(n \leqslant 13)$ and the spectrum is regular since the Coulomb interaction is still dominant in this lower-energy regime. The $n$ mixing starts to occur around $n=15$, and becomes stronger for higher Rydberg series due to the stronger perturbation by the external fields. The inset of Fig. 1 shows the spectrum for the higher energy regime in more detail.

Figures 2(a), 2(b), and 2(d) show a comparison of the calculated photoabsorption spectra (the upper panel of each figure) and the experimental spectra (lower panels), covering the energy range from -400 to $-100 \mathrm{~cm}^{-1}$. [Fig. 2(c) shows the calculated spectrum in the range from -295 to $-215 \mathrm{~cm}^{-1}$ alone, since there are no experimental data available for comparison.] Good agreement is obtained line by line for the whole energy range, including the higherenergy regime well above the Stark saddle point (denoted by $\left.E_{S P}\right)$ in Fig. 2(b), where the SOPT breaks down completely. The small discrepancy in some of the relative intensities can be attributed to the fact that saturation effects occur in the experiment in strong lines, and there exists some uncertainty on the precise value of the magnetic-field strength used in the experiment [1]. The theoretical results in Figs. 1 and 2 are obtained by the use of only 200 pseudospectral radial grid points for each partial wave, and up to 40 partial waves are used to achieve fully converged results. The whole pho-

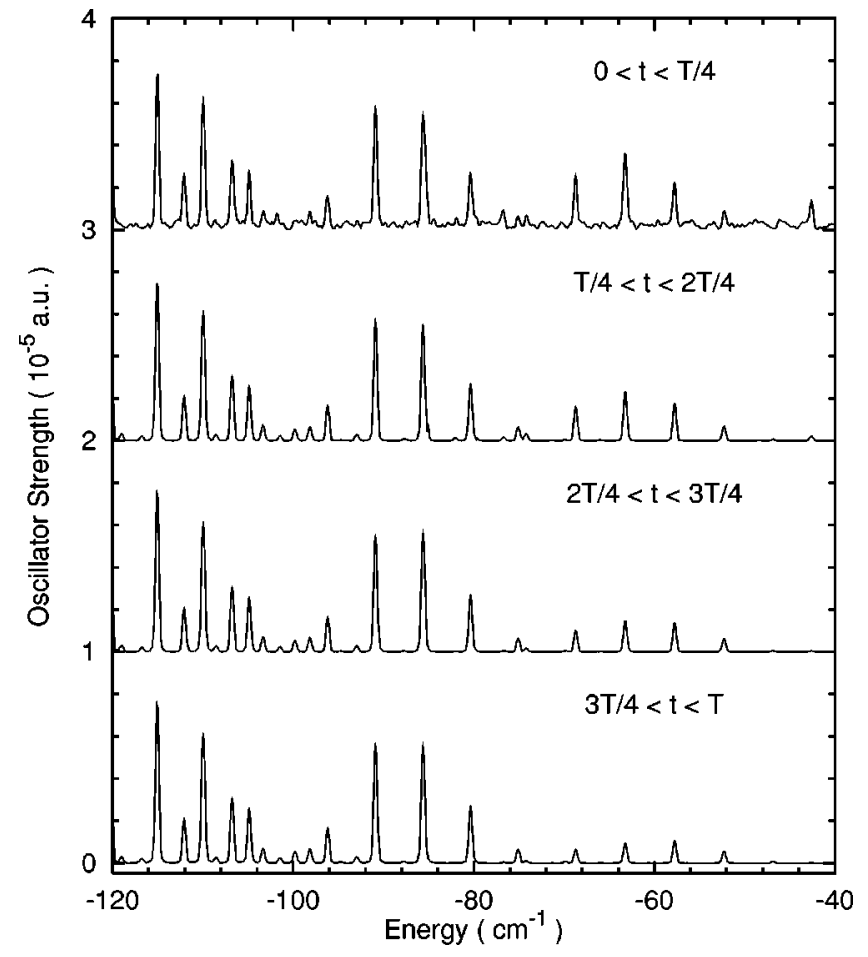

FIG. 3. Theoretical spectra (near the zero-energy threshold) calculated by using different time sections for the Fourier transformation of the time autocorrelation function.

toabsorption spectrum is obtained by a single time propagation of the initial $2 p_{0}$ wave function (times a dipole operator) under the influence of the total Hamiltonian [Eq. (3)], followed by a Fourier transform of the resulting autocorrelation function. To achieve the experimental spectral resolution $\left(0.05 \mathrm{~cm}^{-1}\right)$, we have integrated the time-dependent Schrödinger equation up to $T=3.5 \mathrm{~ns}$, with a time step of the order of 100 a.u. The numerical algorithm is found to be both efficient and stable. To our knowledge, this is the first $a b$ initio time-dependent quantal calculation of the 3D fieldinduced Rydberg atom dynamics and spectrum with spectral accuracy.

One of the most intriguing features in both experimental and calculated spectra is the apparently simpler structure in the higher-energy regime shown in Fig. 2(d), where one would expect to see much denser lines or an order-to-chaos transition (in the classical sense). A clue to the origin of this behavior is revealed by comparing the calculated and experimental spectrum more closely: we notice that our calculated spectrum in the highest energy regime between -120 and $-100 \mathrm{~cm}^{-1}$ (denoted by $A, B, C, D$, and $E$ ) has some extra lines $(B$ and $D)$ not seen in the experimental data. This suggests that $A, C$, and $E$ are associated with longer-living resonance states while $B$ and $D$ are shorter-living states. The experimental data cover a longer time scale than our calculated time span (3.5 ns). Thus while the $B$ and $D$ peaks show up in our calculations, they decay away within the experimental time scale. To support the view of lifetime-dependent photoabsorption spectrum in the higher-energy regime in Fig. 3, we show several different spectra obtained by the Fourier transform of the autocorrelation function $C(t)$ in dif- 
ferent time periods. It is seen that the spectrum obtained from the earliest section $(0<t<T / 4)$ has the largest number of peaks and largest intensities (in the highest energy regime). As time goes by, these peaks decrease in intensity, and some decay away completely before the final propagation time $T$ is reached. From this analysis, we infer that the seemingly simpler structure in the higher energy regime shown in Fig. 2(d) is mainly due to the disappearance (decay) of shorter-living resonances far above the Stark saddle point within the experimental time scale.

Another intriguing phenomenon in both the experimental [1] and theoretical data is that the transition to the ionization regime occurs without any sign of the effect of the Stark saddle point. In fact the results show that ionization does not occur till much higher-energy regime than the Stark saddle energy $E=-2 \sqrt{F}$, which amounts to $-334 \mathrm{~cm}^{-1}$ for $F=2975 \mathrm{~V} / \mathrm{cm}$. We note that the variation in the apparent ionization threshold with magnetic-field strength has also been observed experimentally for the $\mathrm{Rb}$ system [2], and theoretically studied by classical critical point analysis in lower-dimensional systems [25].

In summary, in this Rapid Communication we have presented a general time-dependent approach for accurate, efficient, and long-time propagation of the time-dependent Schrödinger equation, allowing $a b$ initio nonperturbative investigation of the 3D quantum dynamics and high-resolution photoabsorption spectrum of Rydberg $\mathrm{H}$ atoms in crossed fields. The calculated photoabsorption spectra are in good agreement with the experimental data line by line. Further, we have explored the physical origin of several perplexing phenomena in the higher-energy regime observed in the experiment [1].

This work was partially supported by the NSF under Grant No. PHY-9801889. We acknowledge the Kansas Center for Advanced Scientific Computing for the support of the Origin2000 supercomputer time.
[1] G. Wiebucsh et al., Phys. Rev. Lett. 62, 2821 (1989).

[2] G. Raithel et al., Phys. Rev. A 44, 1898 (1991); 47, 419 (1993); 49, 1646 (1994).

[3] J. P. Connerade et al., J. Phys. B 30, 2047 (1997).

[4] E. A. Solovev, Zh. Éksp. Teor. Fiz. 85, 109 (1983) [Sov. Phys. JETP 58, 63 (1983)]; P. A. Braun and E. A. Solovev, ibid. 86, 68 (1984) [ibid. 59, 38 (1983)].

[5] H. Marxer et al., J. Phys. B 27, 4491 (1994).

[6] J. Main and G. Wunner, Phys. Rev. Lett. 69, 586 (1992).

[7] J. P. Connerade, M. S. Zhan, J. Rao, and K. T. Taylor, J. Phys. B 32, 2351 (1999).

[8] J. G. Rao and K. T. Taylor, J. Phys. B 30, 3627 (1997).

[9] J. von Milczewski, D. Farrelly, and T. Uzer, Phys. Rev. A 56, 657 (1997).

[10] T. Uzer et al., Phys. Rev. A 58, 4761 (1998).

[11] H. Friedrich and D. Wintgen, Phys. Rep. 183, 37 (1989).

[12] F. Mignard, Icarus 49, 347 (1982).

[13] M. M. Dignam and J. E. Sipe, Phys. Rev. B 45, 6819 (1992).

[14] A. Bohr and B. R. Mottelson, Nuclear Structure (Benjamin,
Reading, MA, 1995), Vol. II.

[15] G. Mathys, Fundam. Cosm. Phys. 13, 143 (1989).

[16] See, for example, P. Fassbinder et al., Phys. Rev. A 56, 3626 (1997); W. Schweizer and W. Jans, ibid. 57, 1186 (1998).

[17] Time-Dependent Methods for Quantum Dynamics, special issue of Comput. Phys. Commun. 63, 1 (1991), edited by K. Kulander.

[18] X. M. Tong and S. I. Chu, Chem. Phys. 217, 119 (1997).

[19] S.-I. Chu and X. M. Tong, Chem. Phys. Lett. 294, 31 (1998).

[20] E. J. Heller, J. Chem. Phys. 68, 2066 (1978); 68, 3891 (1978).

[21] C. Nessmann and W. P. Reinhardt, Phys. Rev. A 35, 3269 (1987).

[22] W. P. Reinhardt, J. Phys. B 16, L635 (1983).

[23] G. Yao and S. I. Chu, Chem. Phys. Lett. 204, 381 (1993); J. Wang et al., Phys. Rev. A 50, 3208 (1994).

[24] M. R. Hermann and J. A. Fleck, Jr., Phys. Rev. A 38, 6000 (1988); T. F. Jiang and S. I. Chu, ibid. 46, 7322 (1992).

[25] T. Uzer and D. Farrelly, Phys. Rev. A 52, R2501 (1995). 